\title{
pCharacterizing rapid capacity fade and impedance evolution in high rate pulsed discharged lithium iron phosphate cells for complex, high power loads
}

\author{
Derek N. Wong ${ }^{\mathrm{a}}$ \\ derek.wong@mavs.uta.edu
}

David A. Wetz ${ }^{\text {b }}$

wetz@uta.edu

\author{
John M. Heinzel ${ }^{\mathrm{c}}$ \\ john.heinzel@navy.mil
}

Azzam N. Mansour ${ }^{\mathrm{d}}$

azzam.mansour@navy.mil

Phone: 817-272-0719 Fax: 817-272-2253

\author{
${ }^{a}$ Materials Science and Engineering Department, The University of Texas at Arlington \\ 501 West First St, Arlington, TX 76019 \\ ${ }^{\mathrm{b}}$ Electrical Engineering Department, The University of Texas at Arlington \\ 416 Yates St, Arlington, TX 76019 \\ ${ }^{\mathrm{c}}$ Naval Surface Warfare Center, Carderock Division \\ 5001 S. Broad St., Philadelphia, PA, 19112 \\ ${ }^{\mathrm{d}}$ Naval Surface Warfare Center, Carderock Division \\ 9500 MacArthur Blvd., West Bethesda, MD 20817
}

Three26650 $\mathrm{LiFePO}_{4}$ (LFP) cells are cycled using a 40A pulsed charge/discharge profile to study their performance in high rate pulsed applications. This profile is used to simulate naval pulsed power loads planned for deployment aboard future vessels. The LFP cells studied experienced an exponential drop in their usable high-rate recharge capacity within sixty cycles due to a rapid rise in their internal resistance. Differential capacitance shows that the voltage window for charge storage is pushed outside of the recommended voltage cutoff limits. Investigation into the state of health of the electrodes shows minimal loss of active material from the cathode to side reactions. Post-mortem examination of the anodic surface films revealsa large increase in the concentration of reduced salt compounds indicating that the pulsed 
profile creates highlyfavorable conditions for $\mathrm{LiPF}_{6}$ salt to break down into LiF. This film slows the ionic movement at the interface, affecting transfer kinetics, resulting in charge buildup in the bulk anode without successful energy storage. The results indicate that the use of these cells as a power supply for high pulsed power loads is hindered because of ionically resistant film development and not by an increasing rate of active material loss.

Keywords: pulsed power systems; lithium-ion; high rate cycling; electrochemical impedance spectroscopy; surface films

\section{Introduction}

More than ever before, lithium-ion batteries (LIBs) are being considered for deploymentas prime power supplies or as short-term energy storage modules for high power electrical systems[12].Applications range everywhere from simple consumer electronics to advanced electrical loads that will one day be used upon naval ships. In several instances, LIBs may be required to provide capacitor-like performance in which they act more like power dense storage than the energy dense storagethey are traditionally designed to be. The US Navy expects LIBs to be capable of sourcing loads that have unconventionalcyclic demands while still maintaining their recoverable energy storage. As these systems become more prevalent, it is increasingly important to understand the capabilities of LIBs when they are cycled under high rate pulsedprofilesthat are well outside their normal operational range.

The Navy is currently researching a number of applications thatwill require LIBs to both source and sink high power. The charge and discharge modes, respectively, will each last approximately a few secondsand they will be switched repetitively within a single cycle. While some domestic applications demand high rate transient operation from their batteries, none published are equivalent to those being studied by the Navy. The closest would be hybrid electric vehicles (HEVs) that use secondary cells, mainly lithium-ion or nickel metal hydride (NMH)chemistries[3-5], as their primary power supply for driving the electric motors. While the load profile of an HEV requires high power, the rate at which the batteries are operated will nearly always stay well below their maximum ratings so that safety and cycle life for the consumer is maximized.Additionally, the duty cycle of an HEV's transient profile is quite long compared to those the Navy is interested in [4]. In order to meet the size and weight restrictions aboard their mobile platforms, the Navywill require their battery to be operated at itsmanufacturer's maximum allowable rating. Operation near the top of the cell's capabilities stresses them both electrically as well as thermally and introduces new phenomena and challenges that must be understood and overcome. In the Navy'sapplications, safety remains most critical but size and weight of the battery are often considered 
more important than cycle life. If high rate pulsed operationbrings a reduction in cycle life, it is valuable to understand what capacity fade characteristics are unique to it. This need for understanding is what has prompted the work discussed here.

The reader may question why the Navy is pursuing batteries as a possible prime power source for use in high rate applications if their cycle life is significantly reduced. Typically, the Navy has relied upon large diesel powered motor-generator sets to supply a ship's electrical power. As the Navy's electrical requirements increase and their load profiles become more transient in nature, it will become surprisingly more inefficient to use only the ship's motor-generator as a power source. Rapidly ramping on and off a rotational generator's output severely effects its power quality pushing both the voltage and the harmonic distortion well outside of the ratings of the MIL-SPEC-1399 power system standards. On top of the impact to power quality, this type of operation is also fuel inefficient, leading to higher fuel consumption. One proposed solution for improving both efficiency and power quality involves pairing the generator with a hybrid energy storage module (HESM) that utilizes both LIBs and ultra-capacitors to maximize both energy density and power density [6-8]. The ultra-capacitors allow for fast power delivery to the load while the LIBs allow for more pulses from the HESM under a single charge. The HESM is able to act as an augmenting power supply to the motor-generator and as a load to the motor-generator so that a base load can always be maintained.

When sizing the LIB for use within a HESM, several different chemistries can be initially considered however, the unique power profile quickly starts to eliminate many of the available options. Historically, applications utilizing batteries as their prime power source have cycled them under continuous charge and discharge profiles at relatively low to modest rates well below the cells' maximum ratings. These metrics are vastly different from the rapidly transitioning load requirements of interest to the Navy. The energy dense chemistries typically used in consumer electronics, such as $\mathrm{NMH}$ and $\mathrm{LiCoO}_{2}$, are less than ideal for safe and reliable operation within high-pulsed power loads because of the high internal heat generated as well as the high rates of recharge required. These chemistries become unstable at high temperature and the risk/reward is not worth it. A more stable battery chemistry, such as $\mathrm{LiFePO}_{4}$ (LFP), is becoming a popular choice for variable high power loads, however knowledge of their degradation behavior in a high rate pulsed profile is very limited. It is imperative that the degradation behavior of this commercial high power battery chemistry be better characterized under these conditions so that behavior can be predicted and cells can be reengineered to perform under this harsh loading.

A literature search yields very few publications in which the capacity fade ofLFP cellshave been studiedat high rates above their nominal rating [3-6]. Within the bodies of work found in the literature, there is no significant discussion of the aging mechanisms that this type of operation induces within the cells. Instead, the authors present data that shows how capacity fade is accelerated with only 
speculationsas to whyaging occurs from a chemical and materials level perspective. In the work presented here, LFP cell degradation has been characterized at rates of up to $15 \mathrm{C}$ in both continuous and pulsed modes of operation. The cells were studied using both destructive and non-destructive methodsto identify and compare the fundamental mechanisms which lead to reductions in capacity and charge acceptance in high rate continuous and pulsed modes.In this study, $\mathrm{LiFePO}_{4}$ cells of the 26650 form factor are being investigated as a viable cell for use in the Navy's future high-rate, pulsed power systems. The cyclic profile studied here was developedin collaboration with scientists from the US Navy to simulate the types of loads anticipated aboardfuture vessels[9-11].The objective of this study is to evaluate the cell's performance under high rate pulsed conditions and to study how the profile fundamentally degrades the anode and cathode host structures, respectively.

\section{Experiment}

\subsection{Experimental Cell, Test Plan, and Setup}

In the work presented here, four identical commercially available off the shelf lithium-iron-phosphate $\left(\mathrm{LiFePO}_{4}\right)$ cellshave been studied. The anode is composed of graphite using carboxymethyl cellulose and styrene-butadiene rubber as a binder. The cathode is a $\mathrm{LiFePO}_{4}$ bound with polyvinylidene fluoride (PVDF) and mixed with carbon black. The electrolyte is a 1:1:1 mixture of EC:DEC:DMC solvents with $1 \mathrm{M}$ of $\mathrm{LiPF}_{6}$ conducting salts. Three of these cells were cycled using a high rate pulsed profile while the fourth was cycled using a high rate continuous profile. All of the cells used are of the 26650 form factor and the manufacturer's data sheet properties are presented in Table 1.

Table 1: List of specifications for the lithium iron phosphate test cell used in this study.

\begin{tabular}{|c|c|}
\hline 26650 Lithium Iron Phosphate Test Cell \\
\hline Capacity & $2.6 \mathrm{Ahrs}$ \\
Nominal Voltage & $3.2 \mathrm{~V}$ \\
$\begin{array}{c}\text { Charge Cutoff Voltage } \\
\text { (recommended) }\end{array}$ & $3.65 \mathrm{~V}$ \\
$\begin{array}{c}\text { Charge Cutoff Voltage } \\
\text { (maximum) }\end{array}$ & $4.1 \mathrm{~V}$ \\
$\begin{array}{c}\text { Discharge Cutoff Voltage } \\
\text { (recommended) }\end{array}$ \\
$\begin{array}{c}\text { Discharge Cutoff Voltage } \\
\text { (minimum) }\end{array}$ \\
\hline
\end{tabular}




\begin{tabular}{|c|c|}
$\begin{array}{c}\text { Recharge Current } \\
\text { (recommended) }\end{array}$ & $2.6 \mathrm{~A}$ \\
$\begin{array}{c}\text { Recharge Current } \\
\text { (maximum) }\end{array}$ & $5.0 \mathrm{~A}$ \\
$\begin{array}{c}\text { Continuous Discharge } \\
\text { Current (maximum) }\end{array}$ & $42 \mathrm{~A}$ \\
$\begin{array}{c}\text { Pulsed Discharge Current } \\
\text { (recommended for 30 s) }\end{array}$ & $26 \mathrm{~A}$ \\
$\begin{array}{c}\text { Pulsed Discharge Current } \\
\text { (maximum for 30 s) }\end{array}$ & $50 \mathrm{~A}$ \\
\hline
\end{tabular}

Prior to any high rate evaluation, each of the LFP cells studied was cycled five times the nominal $1 \mathrm{C}$ rate (2.6 A) using a constant current (CC) discharge and a constant current-constant voltage (CC-CV) recharge procedure between $2.5 \mathrm{~V}$ and $3.65 \mathrm{~V}$, respectively. Next, a 'baseline' procedure was performed in which the cell was cycled once at the $1 \mathrm{C}$ rate using the same $\mathrm{CC}$ discharge and $\mathrm{CC}-\mathrm{CV}$ recharge procedure used the initial five cycles and each cell'simpedance was measured using electrochemical impedance spectroscopy (EIS). The EIS measurement was made while the cell was at $100 \%$ state of charge (SOC) with an amplitude setting of $10 \mathrm{mV}$ across a frequency range of $0.01 \mathrm{~Hz}$ to $10 \mathrm{kHz}$ recording thirty frequencies per decade. A Princeton Applied PARSTAT® 4000 potentiostat was used to make the EIS measurement. This baseline procedure was repeated after every twenty high rate cycles, discussed next, in order to characterize capacity fade and impedance growth over the cell's cycle life without taking them apart. Once the initial baseline was completed, three of the cells were cycled using a high rate pulsed procedure while a fourth was studied using a high rate continuous procedure. The pulsed procedure is explained in step-by-step form in Table 2. In all experiments, a Maccor industrial cycler was used.The fourth cell in the experiment underwent continuous discharge cycling with a similar procedure to the pulsed cells except for step three. In this cell, step three is replaced by a CC discharge at $15 \mathrm{C}$ similar to the recharge in step four. The voltage limitsin all cells were extended to $2.0 \mathrm{~V}$ in discharge and $4.1 \mathrm{~V}$ in recharge, respectively. The procedure simulates how the battery will be used to augment a generator during discharge and baseload a generator during periods of load inactivity.

Table 2: Cycling procedure, limits, and conditions for the pulsed discharged cells. Continuously discharged cells have a similar procedure, replacing step three with a CC 15C, $40 \mathrm{~A}$ discharge.

\begin{tabular}{|c|c|c|c|}
\hline Step \# & $\begin{array}{c}\text { Step } \\
\text { Name }\end{array}$ & Description & $\begin{array}{c}\text { End } \\
\text { Condition }\end{array}$ \\
\hline \multicolumn{4}{|r|}{$\begin{array}{r}\text { 1C }(2.6 \mathrm{~A}) \text { cycle of CC-CV Charge } \\
\text { to } 3.65 \mathrm{~V}, \text { CC Discharge to } 2.0 \mathrm{~V}\end{array}$} \\
\hline & Baseline Cycles
\end{tabular}




\begin{tabular}{|c|c|c|c|}
\hline 2 & EIS & $\begin{array}{c}90 \text { minute rest, impedance } \\
\text { spectroscopy (10mHz-10kHz, } \\
10 \mathrm{mV} \text { amplitude })\end{array}$ & $\begin{array}{l}\text { End of } \\
\text { Analysis }\end{array}$ \\
\hline 3 & $\begin{array}{l}\text { Pulsed } \\
\text { Discharge }\end{array}$ & $\begin{array}{c}\text { 5s CC Discharge, 1s CC Charge @ } \\
15 \mathrm{C}(40 \mathrm{~A})\end{array}$ & $\begin{array}{l}\text { Cell voltage } \\
\quad \leq 2.0 \mathrm{~V}\end{array}$ \\
\hline 4 & Recharge & CC Recharge@15C (40 A) & $\begin{array}{l}\text { Cell voltage } \\
\leq 4.1 \mathrm{~V}\end{array}$ \\
\hline 5 & Repeat & $\begin{array}{l}20 \text { cycles of Steps } 3 \text { and } 4 \text {. Rest } \\
\text { between each cycle allows cell to } \\
\text { cool to room temperature }\end{array}$ & $\begin{array}{l}20 \text { Cycles } \\
\text { Completed }\end{array}$ \\
\hline
\end{tabular}

A $75^{\circ} \mathrm{C}$ temperature cutoff is programmed into both procedures to limit the thermal stress. The external temperature is monitored by three T-type thermocouples that are sandwiched between two thin layers of polyimide tape and attached to the face of each cell with equal spacing. The polyimidetape provides electrical insulation for the thermocouples from the cell body. The cells are cycledusing the respective high rate procedure until the capacity put back in during the $15 \mathrm{C} \mathrm{CC}$ recharge was $50 \%$ of its original value or until 100 cycles was reached, whichever came first.Afterwards, a final baseline procedure was performed prior to post-mortem analysis. It will be shown later that cells that were pulsed discharged were not able to make it through 100 cycles before the high rate recharge capacity decreased dramatically below $50 \%$.

When evaluating this procedure, the reader should make note of a few conditions imposed. The first concerns the amplitude of the pulsed discharge and recharge currents, respectively. While the discharge current limit is within the manufacturer's maximum ratings, the recharge current is not. Earlier it was mentioned that in future shipboard power systems, both the battery and a motor-generator will supply power to the load and that during short periods of load inactivity, the motor-generator would be baseloaded by the battery. The 5 second discharge period simulates the time when the load is operational while the 1 second high rate recharge period simulates the time when the motor-generator is being baseloaded with the battery acting as the load. The authors realize that the recharge current itself could be a major contributor to aging and degradation and the possible affects are of interest in the research being performed. The second set of conditions to take note of are the minimum discharge cutoff voltage and the maximum recharge cutoff voltage, respectively. Both of these are within the manufacturer's maximum allowable ratings but are of course at the extreme ends. The reason for the extension is to compensate for the large internal voltage rise/drop at 15C. If not extended, the voltage drop/rise would come close to the nominal cutoffs quickly and cause either the discharge or recharge, depending upon the procedure being 
performed, to end prematurely before much of the cell's energy is expended or restored. The widened range provided more time for the charge transfer to occur without actually over-charging or overdischarging the cell. Even though the extended voltage limits are within the maximum values allowed by the manufacturer and do not over-charge or over-discharge the cell in anyway, operation at the extremes could induce accelerated aging similar to that induced when cells are over-discharged or over-recharged [12].The third and final condition to note is the cutoff temperature of $75^{\circ} \mathrm{C}$, which is substantially higher than the $60^{\circ} \mathrm{C}$ limitation recommended by the manufacturer. The limitation was set at $75^{\circ} \mathrm{C}$ so that the depth of discharge (DoD) could be high and not limited by a premature thermal cutoff. It is recognized that the higher thermal stresses will contribute significantly to the aging but these are factors that are of interest to the Navy as they must be able to cycle the cells to high DoD to avoid the battery having to be bigger than necessary to meet the load's capacity requirements. For all the above reasons, it is not intuitively clear how the pulsed nature of the procedure specifically impacts the capacity fade and impedance growth. Thus, the fourth cell which was cycled at the same rate but with a continuous charged/discharge profile includes all of the other voltage and temperature limitations as with the three respective pulsed cells. This way differences in capacity fade can be used to directly compare continuous loading with the unique pulsed system described above.

In addition to the $1 \mathrm{C}$ capacity and EIS measurements, differential capacitance measurements were used to map the charge storage reaction across the voltage range used. This measurement was solved for during several of the $15 \mathrm{C}-\mathrm{CC}$ recharge steps. Differential capacity analysis can illustrate the charge storage process by mapping the derivative charge stored with respect to voltage change. Since the analysis involves looking at very small fluctuations in voltage it is normally performed on a low rate CC step, typically $\mathrm{C} / 25$. To perform this analysis on $15 \mathrm{C}$ recharges, each set of voltage data needed to be filtered to smooth the voltage profile from each of the chosen cycles

\subsection{Electrode Preparation}

Post mortem electrode characterization began by dismantling the cycled cells and analyzing the cathode and anode electrodes using X-ray photoelectron spectroscopy (XPS), X-ray absorption near edge spectroscopy (XANES), and extended X-ray absorption fine structure analysis (EXAFS). Dismantling took place in a glove box filled with argon gas which containedless than 3 ppm of both $\mathrm{H}_{2} \mathrm{O}$ and $\mathrm{O}_{2}$. Samples from both the cathode and anode regions were cut from three regions within the jellyroll including very close to the inner radius, towards the middle of the jellyroll, and close to the outer radius of the jellyroll. Once cut, all of the samples were cleaned in a diethyl carbonate bath for five minutes so that all loose salts would be removed. Once removed from the bath, the electrodes were allowed to dry for ten minutes before being transferred for characterization and chemical analysis. 


\subsection{Characterization}

Two forms of X-ray spectroscopy were utilized to study the post-cycle chemical state of both the anode and cathode electrodes. A synchrotron radiation beamline at Brookhaven National Laboratory (BNL) provided the source for X-ray absorption spectroscopy of the LFP cathode samples (XANES and EXAFS). Using the Fe K-edge energy, the amount of Li lost to side reactions in the cycled cathodes can be quantified. This is accomplished by comparing the edge shift in each of the PD cathodes relative to an LFP powder sample to the edge energy positionsmeasured for $\mathrm{FeO}, \mathrm{FeOOH}$, and $\gamma-\mathrm{Fe}_{2} \mathrm{O}_{3}$ reference samples which each have known oxidation states. Both transmission (TR) and electron yield (EY) detection modes were used to analyze both the bulk and surface LFP particlesrespectively, in the extracted samples. The three sections of the jellyroll, inner, middle, and outer respectively, were analyzed and compared to an LFP foil that was made by spreading LFP powder uniformly on electrically conductive tape. The XANES and EXAFS data analysis was carried out using the IFEFFIT suite of programs named Athena and Artemis, respectively [13,14].

X-ray photoelectron spectroscopy (XPS) was used to identify any compounds that precipitated from side reactions and to quantify their chemical compositions. C 1s, O 1s, F 1s, P 2p, and Li 1s regions were scanned on graphite anodes using a Physical Electronics 5400 X-ray photoelectron spectrometer. The excitation source used was non-monochromatic $\mathrm{Al} \mathrm{K \alpha} \mathrm{X}$-rays located at $54.7^{\circ}$ relative to the analyzer axis operated at $400 \mathrm{~W}(15 \mathrm{kV}$ and $27 \mathrm{~mA})$. The spectra were collected at an electron takeoff angle of $45^{\circ}$ at constant pass energy of $35 \mathrm{eV}$ on a scan area of $1.0 \times 3.5 \mathrm{~mm}^{2}$. Deconvolution using a GaussianLorentzian line shape and a Shirley-type background was conducted to quantify atomic concentrations of each identified compound. The anode spectrums were normalized with respect to the hydrocarbon peak position at $285.0 \mathrm{eV}$ and the cathode spectrums were normalized with respect to carbon black at $284.4 \mathrm{eV}$ $[15,16]$.

\section{Results and Discussion}

\subsection{Electrochemical Observations}

As hinted at earlier, all three of the pulsed discharged (PD)cells were unable to accept charge at 40A after less than fifty cycles of high-rate pulsed operation. A linear fade in the capacity of the $15 \mathrm{C} \mathrm{CC}$ recharge portion of the procedure was measured from all three cells,PD01, PD02, and PD03, respectively, for at least twenty cycles beforea rapid fade was observed. This is opposed to the continuously (CONT) discharged cell that showed linear fade in the $15 \mathrm{C}$ recharge capacity through one-hundred cycles. Though the linear fade was measured through one-hundred cycles, only the data collected from the first sixty 
cycles is shown so that comparison to the three PD cells is clearer. After approximately twenty cycles of linear fade, each of the three respective PD cells rapidly lost the ability to accept recharge current at the $15 \mathrm{C}$ rate. The recharge capacity rapidly decreased to $0 \mathrm{Ah}$ within the next twenty-five and forty-seven cycles, depending on the cell as seen Fig. 1A. Despite each of the three PD cells being unable to accept $15 \mathrm{C}$ recharge, each one was still able to accept and store charge at the $1 \mathrm{C}$ rate. The $1 \mathrm{C}$ baselines performed between every twenty cycles revealed that roughly $6 \%$ capacity fade was measured when compared with the initial $1 \mathrm{C}$ baseline measurement. In contrast, roughly $14 \%$ capacity fade was measured from the CONT cell. This is shown graphically in Fig. 1B.
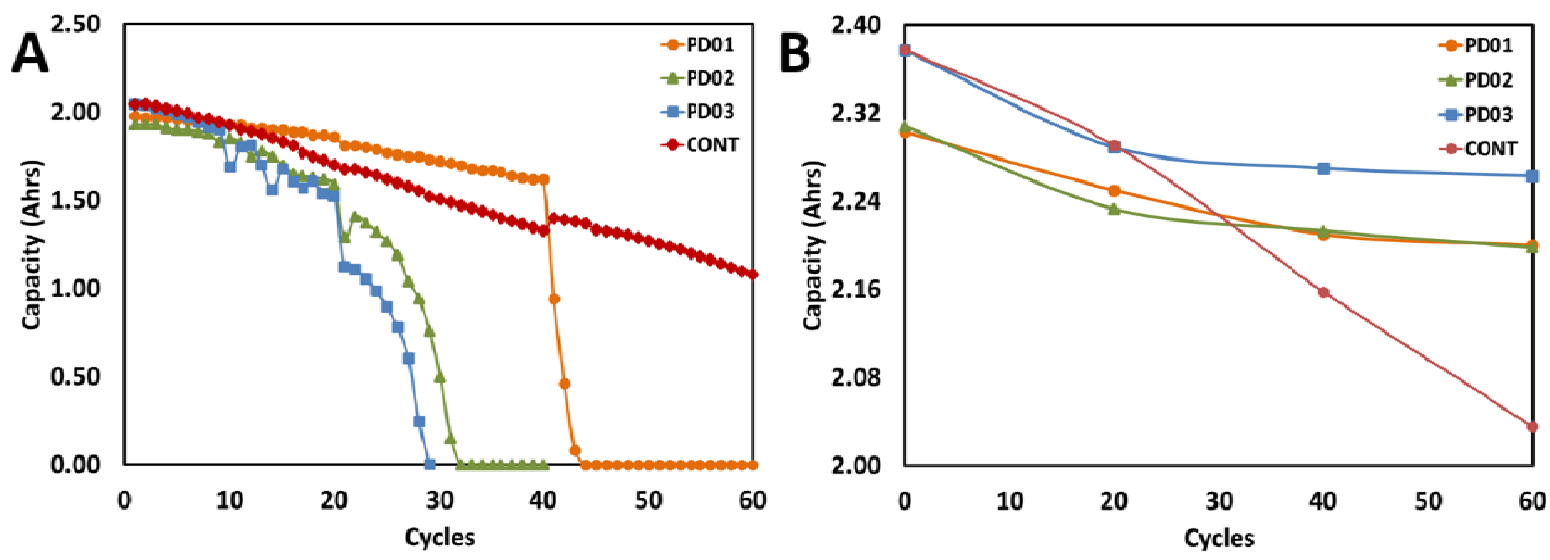

\section{Figure 1: Capacity fade for PD and CONT cells formed using the measured capacities from the A) continuous $15 \mathrm{C}(40 \mathrm{~A})$ recharge step in each cycle and the $\mathrm{B}) 1 \mathrm{C}$ capacities from baselines taken at 20 cycle intervals}

This data suggests there is a critical time at which the charge transfer reaction slowed to the point where the recharge reaction can no longer be completed at the $15 \mathrm{C}$ charge rate. While capacity fade is normally explained by a loss of active Li-ions to side reactions, it is unclear from this data whether or not that is the main mechanism behind the sudden decrease in the PD cells' recharge capacity at $15 \mathrm{C}$. Another possible cause for the sudden inability to accept high-rate recharge is impedance growth which pushes the cell's over-potential above the voltage limitations imposed on the cycler. In short, eventually the overpotential required for the majority of the charge transfer and storage reaction to complete rises above the 4.1V maximum.Differential capacitance spectrums can be used to paint a clearer picture of the evolving charge storage reaction over the life of the cell.

In Fig. 2A, the 15C recharge differential capacity curves from cell PD 01 of cycles 1, 20, and 40, respectively,are shown. A gradual rise in charge transfer resistance is seen before the drop in charge acceptance thatbeginsduring cycle 41 . Early in the life of the cell, the majority of charge storageoccurred 
when the cell's voltage was between $3.65 \mathrm{~V}$ and $4.1 \mathrm{~V}$. Comparison of the differential charge profilesfrom cycles 1,20 , and 40 , respectively, show that there is a gradual increase in the charge storage activation voltage which is the voltage at which successful charge storage begins. Over the first forty cycles of cell PD01, the differential charge profiles were nearly identical with respect to their curvatures; however, the curves are compressed within a smaller voltage range as more 15C PD cycles are completed.The area under each of the curves, which represents the total charge stored in the cellduring a cycle, reduced at a steady rate. This mirrors the trendseen in the capacity fade profile for the first forty cycles of cell PD01.On the other hand, the differential profilesof cycles 41 to 43 , which are shown in Fig. 2B, show that there is a dramatic reduction in the amount charge stored within the operating voltage range of $2.5 \mathrm{~V}$ to 4.1 V. From cycle 41 to 43 , thecharge storage time is shortened such that it occurs within a range of less than $100 \mathrm{mV}$. This indicatesthat there is an abrupt increase in the cell's internal resistance. This is most clearly seen when comparing the differential curves from Fig. 2A to those in Fig. 2B. Between cycles 40 and 41 , the activation voltage shifted to the point where the majority of the profile falls outside of the $3.65 \mathrm{~V}$ to 4.1 Vrange. However, the magnitude and curvature of the differential curves are still similar to those of cycles 1,20 , and 40 , respectively. It can be inferred that if the voltage were not limited to $4.1 \mathrm{~V}$, the charge storage may still be successful at the $15 \mathrm{C}$ rate. Overall, the differential capacitance analysesandcapacity fademeasurementsshow that there was an abrupt slowing of the kinetics of the charge storage reaction due to a rapid rise in internal cell resistance. This rise prevented all three respective PD cells from accepting charge at the $15 \mathrm{C}$ rate within the $2.0 \mathrm{~V}$ to $4.1 \mathrm{~V}$ range in which they were cycled.
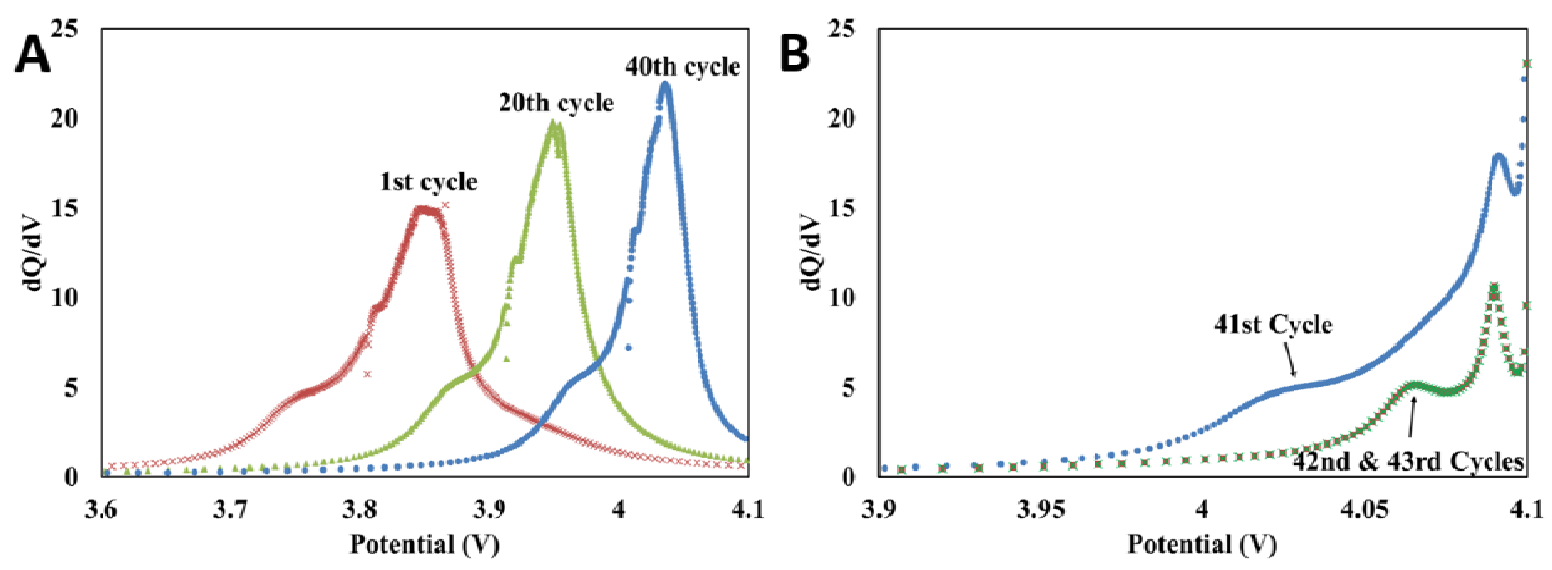

Figure 2: Differential capacitance spectrums derived from the $\mathrm{CC}$ 15C recharges of cell PD01 at A) cycles 1,20 , and 40 . Separate spectrums were derived from the same step at cycles B) 41, 42, and 43 during the rapid capacity decline. 
Once it was clear that a rise in the internal impedance had occurred, it was necessary to understand what had caused the impedance to grow. Analysis of the electrochemical impedance spectroscopy (EIS) results at each of each twenty cycle interval was performed to characterize the evolution of cell impedance. The EISmeasurements made from allthree respective PD cellsare shown in Fig. 3A through Fig. $3 \mathrm{C}$. In the data, there is a clear separation of the mid-frequency semi-circle, located within in the 8 $\mathrm{m} \Omega$ to $17 \mathrm{~m} \Omega$ range, from one distinct semi-circle into two. The first semi-circle remains within the same real impedance range as the original spectrum did, while the secondappears in the $17 \mathrm{~m} \Omega$ to $28 \mathrm{~m} \Omega$ range with roughly the same magnitude of imaginary impedance. When consulting the literature, many have attributed these mid-frequency semicircles to be a combination of three impedance elements including the charge transfer impedance, impedance of the SEI layer, and the bulk electrode impedances [17-19]. Since the bulk electrode impedance does not normally evolve much over cycle life, it is most likely that the separationis caused by a rapid growthof the SEI layer impedance and/or from rise inthe charge transfer impedancein the cell. The charge transfer impedance represents the ease of which ions can be exchanged through the double layer at each electrode interface. The efficiency of the charge transfer process is influenced by several factors, the most important being the contact area with the electrolyte at each electrode. If the contact area were reduced and the rate of ion transfer through the double layer declined, it would correspond to a decrease in that cell's ability to transfer Li-ions from the electrolyte to the electrode. 

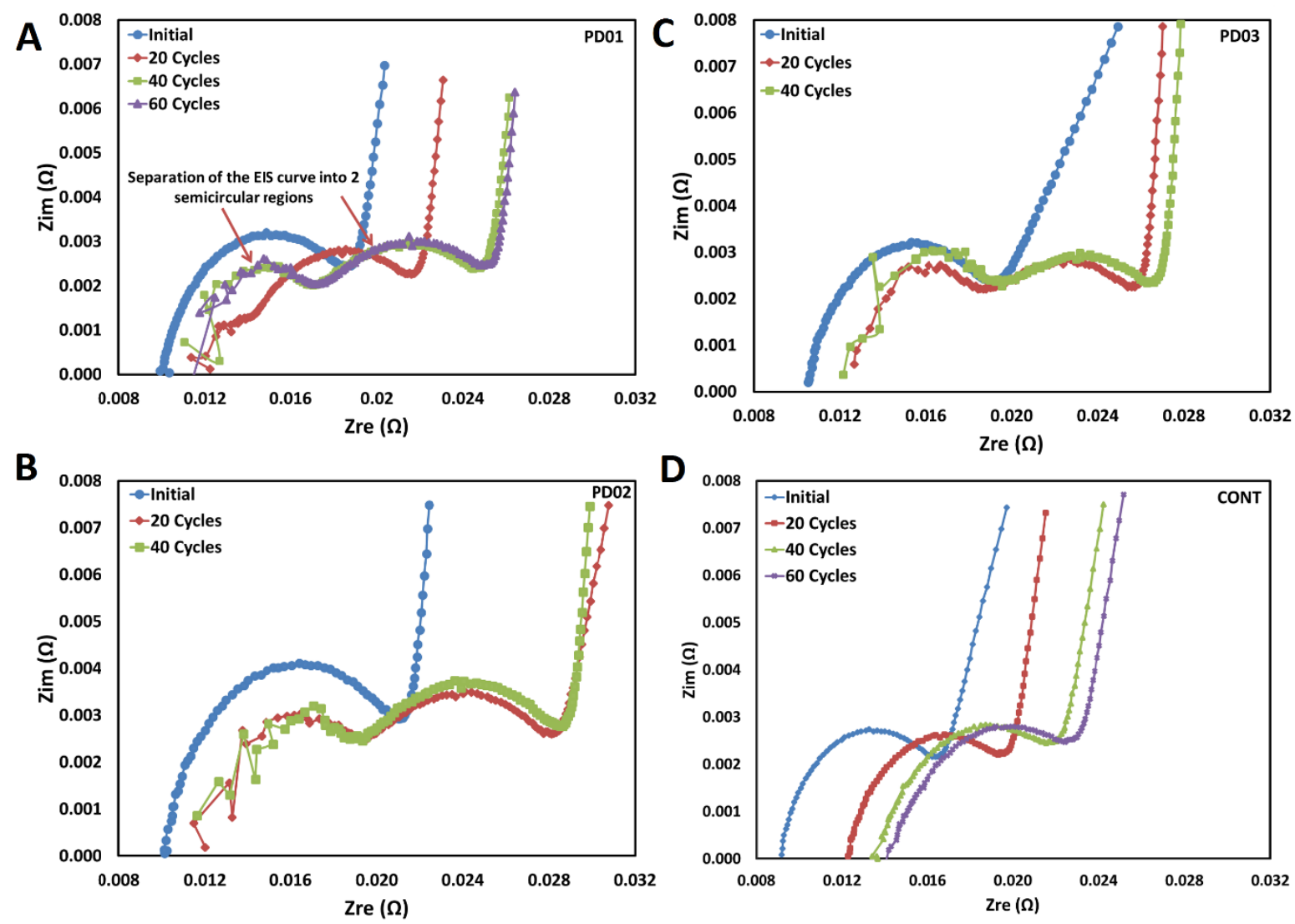

\section{Figure 3: EIS spectrums collected every 20 cycles for cells A) PD01, B) PD02, C) PD03, and D) CONT. The pulsed discharged cells show a separation of the mid-frequency semi-circle.}

It's also equally possible that this separation in the PD cells was the result of an SEI filmwhich most likely forms at the graphite anode-electrolyte interface. This has been discussed by previous researchers who have concurred that SEI layer growth is a byproduct of the side reactions associated with the nonspontaneous charging process in LIBs [20-22]. Normally, as the electrolyte solution reacts with active Liions to form the SEI layer, other byproducts, such as polyethylene oxide (PEO), form between the SEI and the graphite anode through electrolyte co-intercalation, which increases the interfacial contact area between the SEI and graphite as the film grows. This can result in a sharp decrease in charge transfer resistance and a slight increase in the double layer's capacitance [23]. Therefore, the formation of an SEI can actually provide stability and in some cases protect the anode material underneath from further side reactions with the electrolyte. However, if there is a persistent growth of an ionically resistant film, then the SEI formed would act as more of a barrier to Li-ion transfer instead of providing stability[17]. While these 2-electrode results can't validate these theories alone, it has shown that one of these three impedance elements discussed evolves during PD cycling to affect the low frequency response of the cells. Low frequency response is correlated to diffusional phenomenon such as the movement of active Li-ions through mediums such as the SEI. As stated, particular chemical compounds which comprise the 
SEI can adversely affect the rate of diffusionof $\mathrm{Li}$-ions into the anode. Therefore, it is imperative that the individual SEI compounds of all of the test cells be identified. Additionally, it is equally important to determine which side reactions are specifically forming these compounds in order to define the source of the degradation.

When studying the CONT cell, the impedance spectrum, shown in Fig. 3D, shifts along the real axis while having little increase in the imaginary scale. Notice however, that there is no separation of the mid frequency semi-circles into two as was seen in the PD cells. The magnitude of the shift decreases in between each of the twenty cycle intervals. Physically, this is attributed to the gradual development of the SEI layer which, as mentioned, can stabilize and protect the electrode surface from further side reactions with the electrolyte. This gradual increase in the cell's real impedance is typical of the type of evolution found in literature of studies done on impedance evolution of LIBs [17,18,23]. This means that in the CONT cell, the SEI layer was not able to affect the low frequency, diffusional properties of the cell in a way that drastically influenced its impedance profile as clearly occurred in all the PD cells. Most importantly, the drastic difference in the effect of the low frequency region shows the CONT cell degraded in an entirely different manner compared to the PD cells.Also, the CONT cell impedance spectrum provides a reference for the type of evolution typically seen in this cell when operating at $15 \mathrm{C}$ rates. When discussing the causes behind the difference in impedance evolution, it is reasonable to suggest that the separation seen in the PD cells is not only a result of the high cycling rate, increased cutoff voltages imposed, or high thermal stress. Thus, the major difference between the PD cells and the CONT cell which could explain this difference in impedance evolution is the profile under which each cell was cycled.

\subsection{Ex-Situ Electrode Material Analysis}

Extracted electrodes studied using XANES, EXAFS, and XPS techniques yielded valuable information on their chemical state. XANES and EXAFS revealed information about the concentration of lithium in cycled LFP cathode particles. XPS identified the surface compounds in films on cycled electrodes and determined their concentrations. The data collected from these methods were used to contrast the differences in Li concentration, atomic order, and surface chemistry between PD and CONT cycled cathodes. These differences are key to understanding which degradation mechanisms contribute to the capacity fade in each case.

XANES analysis showed little difference in the oxidation state and atomic order between PD cathodes and a reference LFP foil. Cathodes extracted from cells PD01, PD02, and PD03 showed a 0.69 $\mathrm{eV}, 0.52 \mathrm{eV}$, and $0.4 \mathrm{eV}$ shift respectively in their Fe edge energies relative to the edge energy measured 
in the LFP foil. This is illustrated in detail in Fig. 4 which also revealsno variability between the sampled cathode sections with regards to the edge energy or curve profile. Any delithiated LFP particles populated byFe ${ }^{3+}$, like in $\mathrm{FePO}_{4}$ rich particles, would yield a positive shift in the edge energy from its lithiated $\mathrm{Fe}^{2+}$ position due to the increased energy required to remove an electron from its orbital [24].However, the small shifts in edge energies of the PD cathodes suggests the abundance of particles in these three cells still exists in the $\mathrm{Fe}^{2+}$ oxidation state, meaning they still are bonded with active Li. By this evidence, a loss of active Li would not explain the rapid capacity fade in PD cells even thoughit is known thatLi-ion cells which arecycled at rates well above their nominal rate also have a greaterdegradation rate. When utilizing surface sensitive EY detection, the magnitude ofedge shifts was even smaller with a maximum of $0.12 \mathrm{eV}$ indicating almost complete lithiation of particles near the electrolyte-cathode interface. These results were further supported by EXAFS Fourier transforms which revealed an equally unsubstantial change in the atomic order of the PD cathodes.
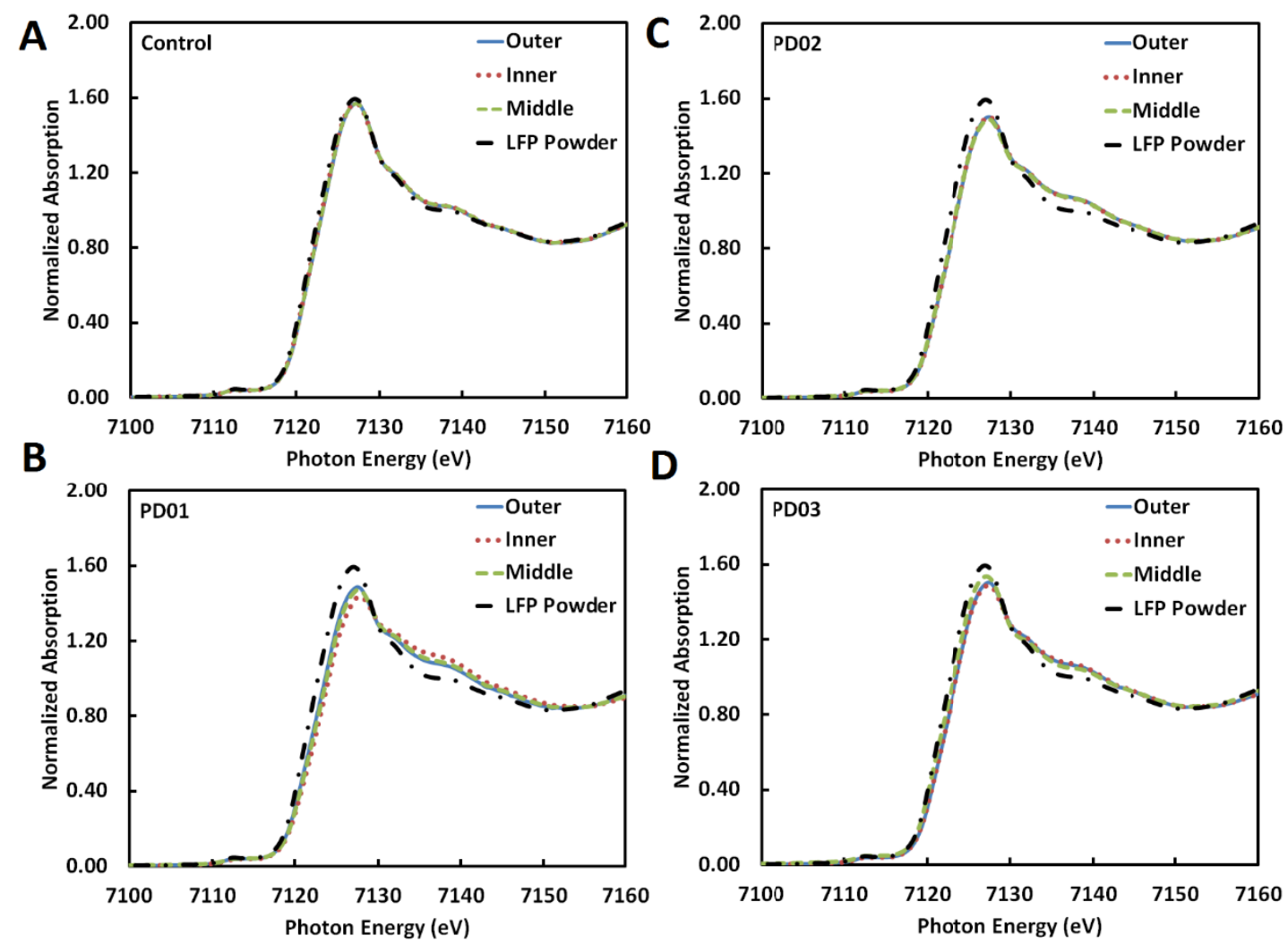

Figure 4: XANES transmission spectrums of cathodes extracted from three sections of the roll for a A) control cell and cells B) PD01, C) PD02, and D) PD03. A spectrum collected from an LFP powder sample is shown as a dashed line on each plot as a reference.

The EXAFS spectrums, shownin Fig. 5, revealed that the atomic order in PD cathodes closely resembled the LFP foil and control. There was an increase in the signal from atoms at a spacing of 3.4 
Angstroms which is the Fe-Fe interatomic distance of olivine structured LFP.This indicates that the olivine crystal structure is still intact in the majority of particles for PD cathodes.Across the EXAFS spectrum, the differences seen in PD cathodes are subtle in comparison to the LFP foil which indicate no large change in order of the atoms. Most notably, the position of the Fe-O peak at $1.5 \AA$ doesn't shift again indicating that the degree of lithiation is unchanged. In their study, Hong et al presented the breakdown of this peak into the separate bond distances represented. As the degree of lithiation decreased, the peak shifted towards a lower spacing distance indicating its transition towards pure $\mathrm{FePO}_{4}$ [25].However, no such shift was observed in the 1.5 ̊ peak from EXAFS spectrums of the PD cathodes. All in all, EXAFS revealed that post-cycleatomic order of the three PD cathodes were not only identical to each other, but also nearly unchanged compared to the LFP foil respectively. Thisdemonstrated consistency in the final chemical state between all three PD cathodes and reinforced that therapid capacity fade didn't stem from active material loss.
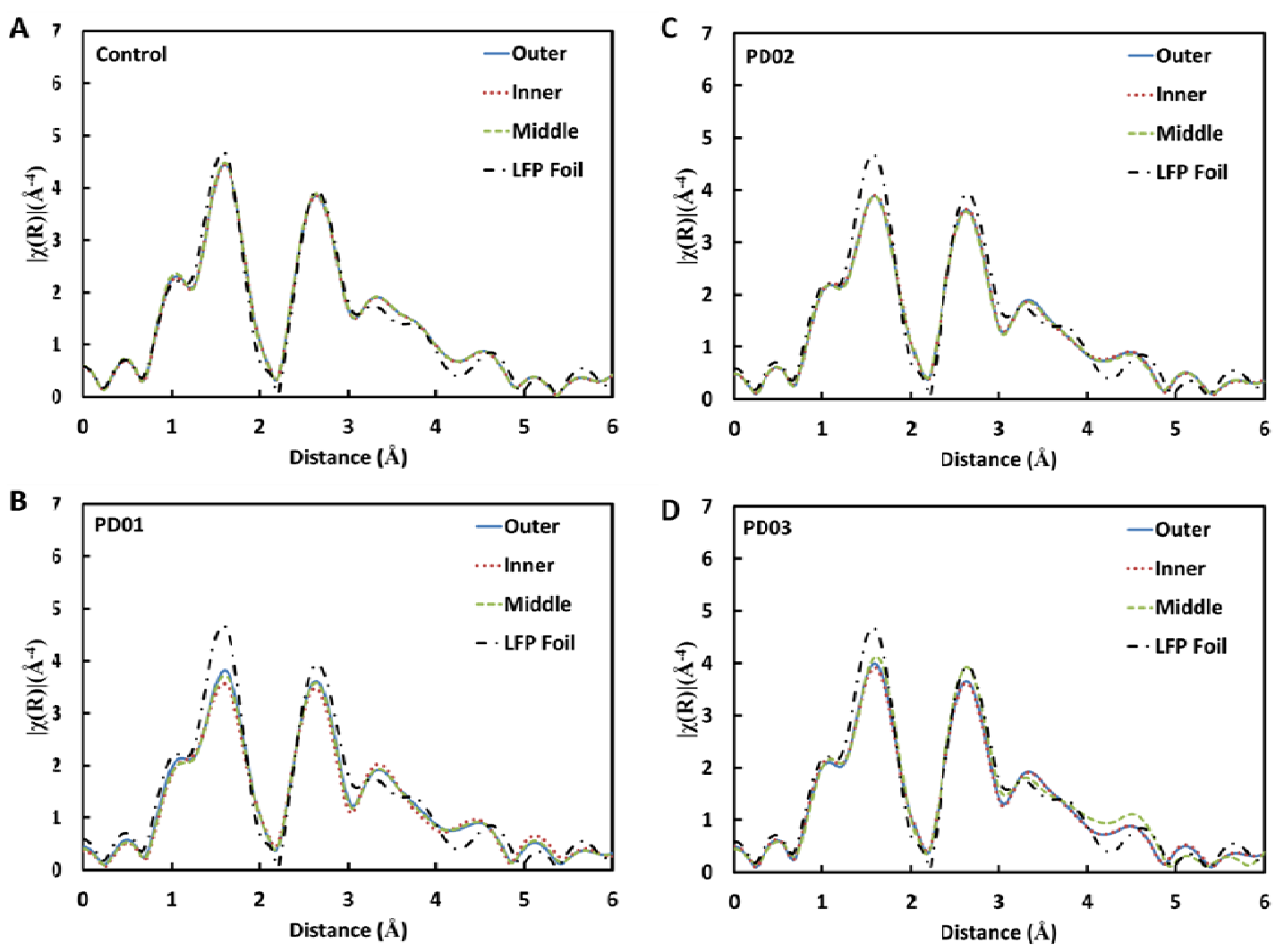

Figure 5: EXAFS spectrums generated from the XAS data with a $k^{3}$-weight and a range of 3.0-10.5 $\AA^{-1}$. The same piece of foil made from LFP powder presented in the XANES spectrums is shown again here as a dashed line as a reference.

To quantify the relative amount of $\mathrm{Fe}^{2+}$ in $\mathrm{PD}$ cathodes and the control, the edge shift calculated between the LFP foil and each PD cell were compared to thedifferences in edge energy between reference 
samples of known oxidation states. Data shown in Fig. 6 was calculated by comparing the PD cell edge shifts to the $4.52 \mathrm{eV}$ per unit change in valency obtained by measuring the edge positions of $\mathrm{FeO}$, $\mathrm{FeOOH}$, and $\gamma-\mathrm{Fe}_{2} \mathrm{O}_{3}$. The magnitude of the edge difference between each of the reference samples illustrated a linear correlation between oxidation states of $\mathrm{Fe}^{2+}, \mathrm{Fe}^{3+}$ and $\mathrm{Fe}^{4+}$. By use of this method it wasrevealed that cathode samples from cells PD01, PD02, and PD03 had $\mathrm{Fe}^{2+}$ concentrations of $85 \%$, $88 \%$, and 91\% respectively when using edge shifts taken from TR data. With EY detection data, PD01, PD02, and PD03 were calculated to be populated by $97 \%, 100 \%$, and $100 \%$ of $\mathrm{Fe}^{2+}$ phases. From this it clear that there exists a differential in the concentrationFe ${ }^{2+}$ phases between the bulk and surface LFP particlesin all of the three PD cathodes. The PD profile used in this study may explain this phenomenon asit creates a localization of $\mathrm{Li}$-ions at the electrolyte-anode interface due to the repetitive reversal of ion flow. Also, it's been shown that the number of active intercalating LFPparticles and their homogeneity across the interface is highly dependent on the electrochemical cycling conditions, including rate[26,27].In the end, the quantitative XAS data from all three PD cathodes didn't suggest there was an accelerated loss of active material on the surface or the bulk LFP particles based on the concentration of $\mathrm{Fe}^{2+}$ phases. It is logical to infer that if the capacity fade in PD cells wasn't a result of active material loss, then it is instead a physical component, which impedes the intercalation of Li-ions. Therefore, identifying the surface film compounds are critical in discovering the degradation mechanism in the PD cells.

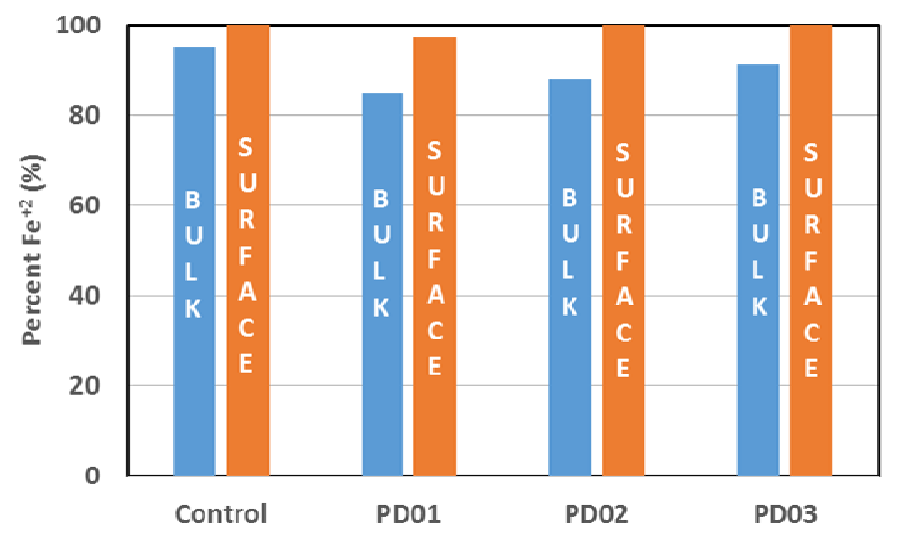

Figure 6: $\mathrm{Fe}^{+2}$ concentration in pulsed discharged cathodes derived by comparing their edge shifts to $\mathrm{Fe}_{2} \mathrm{O}_{3}, \mathrm{FeOOH}$ and $\gamma-\mathrm{Fe}_{2} \mathrm{O}_{3}$ reference spectra. Edge shifts are based on edge energies as measured at half-height.

XPS conducted on extracted graphite anodes revealed keycompositional differences between surface films formed onPD cells and those formed onthe CONT cell. In the C 1s region, seen in Fig.7, the peak assigned to $\mathrm{Li}_{2} \mathrm{CO}_{3}(290.36 \mathrm{eV})$, which is a common SEI compound, was more intense in the CONTanode spectrum than the same peak in spectrums collected from PD01 and PD02 respectively. 
Surprisingly, the C 1s spectrums of anodes from PD01 and PD02 closely resembled thespectrum from an anode extracted from a fresh, uncycled LFP cell of the same batch.

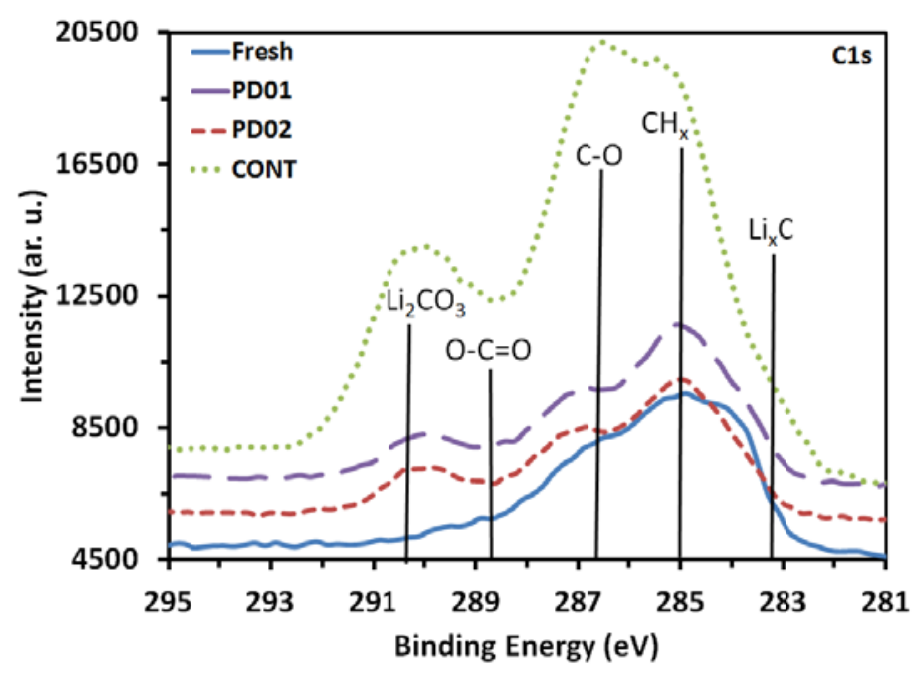

Figure 7: XPS spectrums from the $\mathrm{C}$ 1s region of graphite anodes extracted from a fresh cell, PD01, PD02, and CONT. Deconvoluted peaks are marked and identified at their corresponding binding energies. PD03 was not included to avoid crowding but shows the same trend as the other two PD cells.

Deconvolution of the peaks in the $\mathrm{C} 1 \mathrm{~s}$ regionquantified the $\mathrm{Li}_{2} \mathrm{CO}_{3}$ concentrations in the surface films of each cell as 3.0 at. $\%$, and 2.9 at. $\%, 5.1$ at. $\%$ of the total concentration of compounds onPD01, PD02, and CONT anode surface films, respectively.In linearly degrading cells, like the CONT, $\mathrm{Li}_{2} \mathrm{CO}_{3}$ concentration increases with cycling as it is a common electrolyte decomposition product via the reaction:

$$
\text { Ethylene carbonate }+2 \mathrm{e}^{-}+2 \mathrm{Li}^{+} \rightarrow \mathrm{Li}_{2} \mathrm{CO}_{3}+\mathrm{CH}_{2}=\mathrm{CH}_{2}
$$

Additionally, the signal from the peak at $286.7 \mathrm{eV}$, assigned to C-O single bonds stemmingfrom nonstoichiometric side reactions, would also intensify. The concentration of C-O bonds detected on PD01 and PD02 anodes were 5.6\%and 5.2\% respectively in contrast to $11.02 \%$ detected on the CONT anode. In the $\mathrm{O} 1 \mathrm{~s}$ region, shown in Fig.8A, the amount of oxygen detected that was assigned to $\mathrm{Li}_{2} \mathrm{CO}_{3}$ or $\mathrm{C}=\mathrm{O}$ bonds at $531.9 \mathrm{eVwas} 14.0$ at. $\%, 15.8$ at. \%, and 23.3 at. \% of the surface compounds in PD01, PD02, and CONT anode surface films respectively. It's interesting that the concentration of $\mathrm{Li}_{2} \mathrm{CO}_{3}$ is greater inthe CONT anode surface film yet it continued to store charge at $15 \mathrm{C}$. The F 1s, P 2p, and Li 1sspectrums, seen in Fig.8B-D, showed far greater intensity in thepeak assigned to LiF at 685.5 eVon PD01 and PD02 anode films compared to the CONT film. From deconvolution of the F 1s spectrum, the LiF concentration in PD01 and PD02 surface filmswere22.5 and 23.0 at.\% respectively, while only 5.3 at.\% in the CONT film. This was the greatest differential in concentration detected of a single surface film compound 
between the PD and CONT films.It's been documented that metastable products from side reactions like $\mathrm{Li}_{x} \mathrm{PF}_{\mathrm{y}}, \mathrm{Li}_{\mathrm{x}} \mathrm{PF}_{\mathrm{y}} \mathrm{O}_{\mathrm{z}}$, and $\mathrm{Li}_{2} \mathrm{CO}_{3}$ can decompose further by reacting with impurities in the cell to form $\mathrm{LiF}$, which is an ionically resistive compound[28,29].Clearly, the quantitative XPS analysis shows that in the early development of the surface films on PD01 and PD02 anodes the conditions were more favorable for the formation of LiF while the CONT cell developed films rich in reduced electrolyte compounds like $\mathrm{Li}_{2} \mathrm{CO}_{3}$. Regardless of which specific reactions are taking place $\mathrm{LiF}$, an ionically impeding compound,was clearly more favorably precipitated in these 26650 LFP cells when the Navy's high power pulsed loading was being simulated.The repetitive reversal of the direction of Li-ion flow may provide more favorable conditions for the $\mathrm{LiF}$ producing side reactions to occur at the anode surface during PD.In conclusion, XPS has shown that pulsed discharged cells developed surface films that contained high concentrations of LiFwhich leda dramatic increase in the low frequency impedanceresulting in arapid capacity fade at $40 \mathrm{~A}$ charging rates.
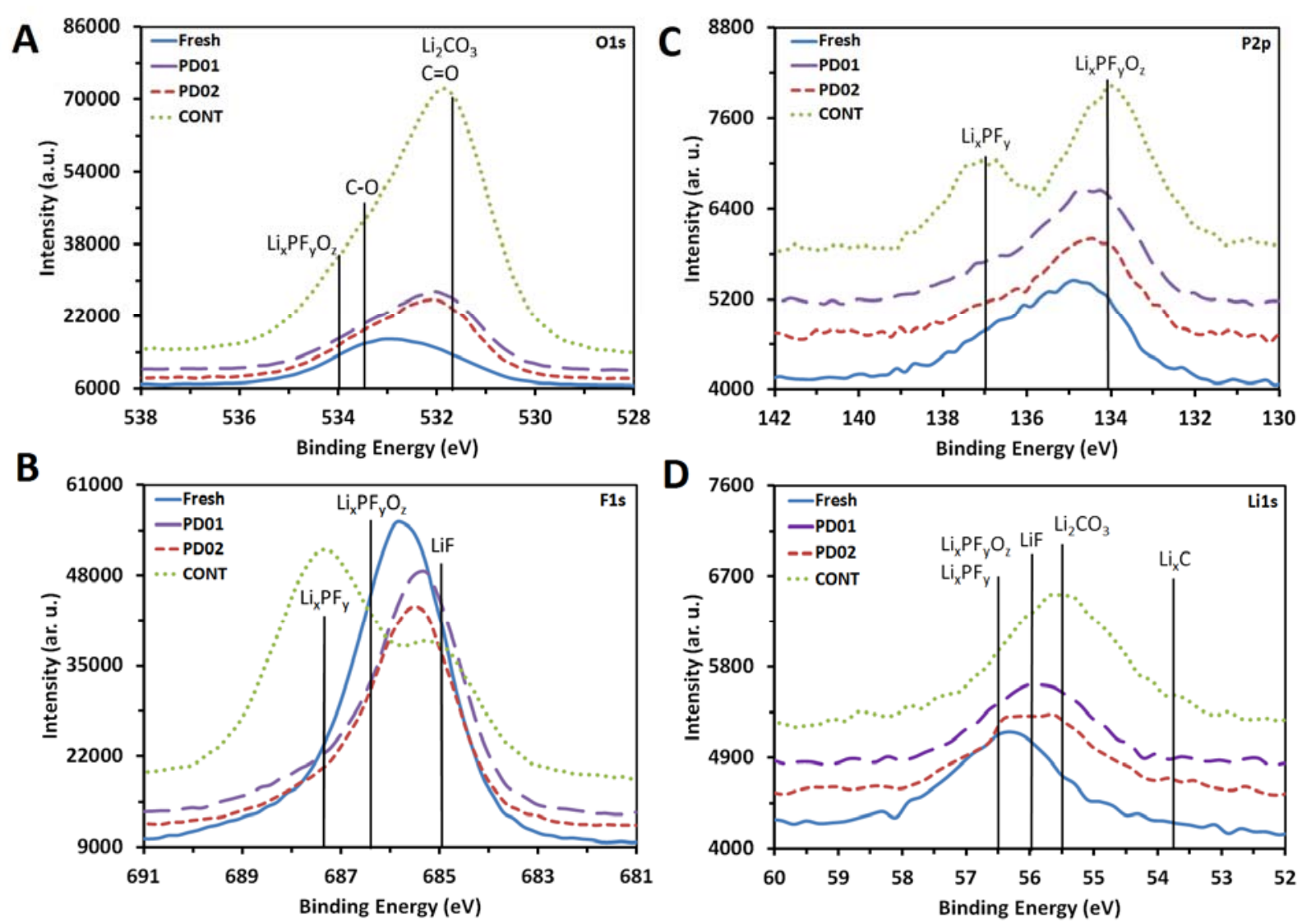

Figure 8: XPS spectrums of graphite anodes in A) O1s, B) F1s, C) P2p, and D) Li1s regions from a fresh cell, PD01, PD02, and CONT. Deconvoluted peaks are marked and identified at their corresponding binding energies. 
The composition of the surface films developed on PD anodeshave a strong contrast to those on continuously discharged anodes. More importantly, the results of this study indicated that the pulsed discharge profile was key in inducing this contrast since other cycling parameters were identical in the continuously discharged cell. The reader'sclaim may be that reduction of conducting salts is more favorable under extreme thermal conditions which may produce these results[30]. However, as mentioned, the cycler limits the outer temperature and the cells are allowed to cool to room temperature between each cycle. Also, the thermal data reveals there is less than $3^{\circ} \mathrm{C}$ difference in the maximum external temperature between all cells including the continuously cycled cell. Thus, the thermal stress is similar between all the cells in this experiment. It is notable to mention that there's been ongoing research into manipulating the concentration of the formed $\mathrm{SEI}$ by introducing $\mathrm{Li}_{2} \mathrm{CO}_{3}$ as an additive in electrolyte solutions[31]. The addition of $\mathrm{Li}_{2} \mathrm{CO}_{3}$ has been shown to reduce gas production andaides in the formation of a passive film. However, it is very unclear whether the addition of $\mathrm{Li}_{2} \mathrm{CO}_{3}$ would prevent the same result from reoccurring under high rate pulsed loading.

\section{Conclusion}

Three of thecommercial 26650 LFP secondary cells which were cycled under a 5 second discharge, 1 second recharge, $40 \mathrm{~A}$ pulsed dischargeprofile exhibited rapid fade of their $40 \mathrm{~A}$ recharge capacity to 0 Ahrs within 60 cycles while a fourth cell experienced linear fade through 100 cycles of operation in a 40 A continuously discharged operation. Impedance spectroscopy revealed separation of the mid-frequency semi-circle in the Nyquist spectrums of the three PD cells indicating an abrupt rise in the internal impedance of diffusional processes. At the end of testing, charge storagewas impeded to the point where high rate recharging at 40A was no longer possible in these PD cells. Furthermore, XANES analysis on post-cycledelectrodes revealed that at least $85 \%$ of particles in pulsed discharged cathodes remained in the $\mathrm{Fe}^{2+}$ oxidation state, indicating that plenty of active material was still available despite the lack of successful charge storage at 40 A. EXAFS detected no substantial changes in the atomic order of the PD cathodes.XPS revealed that PDanodesdeveloped SEIfilms with 4 times theatomic concentrationof LiFstemming from increased amount of $\mathrm{LiPF}_{6}$ saltreduction. Concentrations of electrolyte decomposition compounds, likeLi $\mathrm{CO}_{3}, \mathrm{C}=\mathrm{O}$ and $\mathrm{C}-\mathrm{O}$,were farlowerin surface films on anodes of PD cells. In fact,XPS spectrums of PD anodes in the C 1s, O 1s, P 2p, F 1s, and Li 1s regionsclosely resembled spectrums of a freshanode extracted from an uncycled, fresh control cell. The conclusion drawn from comparing chemical analysis data between the pulsed and continuously discharged electrodes is that the conditions for forming LiF were more favorable in pulsed discharged cells during the early stages of surface film development.This caused the internal impedance of the pulsed discharged cells to rise rapidly, eventually extinguishing any successful recharge storage at the 40 A rate. Thus, if LIBs are to be used as a pulsed 
power source in future US Navy applications, this issue must be addressed on an individual cell level in order to avoid oversizing the modules, especially for systems aboard mobile platforms.

\section{Acknowledgements}

The authors would like to thank the Office of Naval Research for its support through grant N00014-11-10659. Any findings and opinions are those of the authors and not necessarily those of the Office of Naval Research.The XAS experiments were conducted at the National Synchrotron Light Source of Brookhaven National Laboratory, which is supported by the U.S. Department of Energy, Office of Basic Energy Sciences, under contract no. DE-AC02-98CH10886.

\section{References}

[1] B. Scrosati and J. Garche, "Lithium batteries: Status, prospects and future," Journal of Power Sources, vol. 195, no. 9, pp. 2419-2430, 2010.

[2] D. Wetz, P. Novak, B. Shrestha, J. Heinzel and a. S. Donahue, "Electrochemical Energy Storage Devices in Pulsed Power," IEEE Transactions on Plasma Science, vol. 10, no. 2, p. 3034 - 3042, 2014.

[3] L. Lam and R. Louey, "Development of ultra-battery for hybrid electric vehicle applications," Journal of Power Sources, vol. 158, no. 2, pp. 1140-1148, 25 August 2006.

[4] O. Tremblay, "A Generic Battery Model for the Dynamic Simulation of Hybrid Electric Vehicles," in Vehicle Power and Propulsion Conference, Arlington, TX, 2007.

[5] S. Peterson, J. Whitacre and J. Apt, "The economics of using plug-in hybrid electric vehicle battery packs for grid storage," Journal of Power Sources, vol. 195, no. 8, pp. 2377-2384, 2010.

[6] J. Zheng, T. Jow and M. Ding, "Hybrid power sources for pulsed current applications," IEEE Transactions on Aerospace and Electronic Systems, vol. 37, no. 1, pp. 288-292, 2002.

[7] I. Cohen, D. Wetz and J. Kelley, "Evaluation of a Hybrid Energy Storage Module for Pulsed Power Applications," IEEE Transactions on Plasma Science, vol. 42, no. 10, pp. 2948-2955, 2014.

[8] I. Cohen, C. Westenhover and D. Wetz, "Evaluation of an actively contolled battery-capacitor hybrid energy storage module (HESM) for use in driving pulsed power applications," in Pulsed Power Conference, Austin, TX, 2015.

[9] J. Mankowski, J. Dickens, M. Giesselmann and B. McDaniel, "A Bench Top Railgun With Distributed Energy Sources," IEEE Transactions on Magnetics, vol. 43, no. 1, pp. 167-169, 2007.

[10] H. Fair, "Progress in Electromagnetic Launch Science and Technology," IEEE Transactions on Magnetics, vol. 43, no. 1, pp. 93-98, 2007. 
[11] J. McFarland and I. McNab, "A long-range naval railgun," IEEE Transactions on Magnetics, vol. 39, no. 1, pp. 289-294, 2003.

[12] S. Yang, Y. Song and P. Y. Zavalij, "Reactivity, stability and electrochemical behavior of lithium iron phosphates," Electrochemistry Communications, vol. 4, no. 3, pp. 239-244, 2002.

[13] M. Newville, "IFEFFIT: Interactive EXAFS analysis and FEFF fitting," Journal of Synchrotron Radiation, vol. 8, no. 1, pp. 322-324, 2001.

[14] B. Ravel and M. Newville, "Athena, Artemis, Hephaestus data analysis for $x$-ray absorption spectroscopy using IFEFFIT," Journal of Synchrotron Radiation, vol. 12, no. 1, pp. 537-541, 2005.

[15] M. Morcrette, Y. Chabre and G. Vaughan, "In-situ X-ray diffraction techniques as a powerful tool to study battery electrode materials," Electrochimica Acta, vol. 47, no. 1, pp. 3137-3149, 2002.

[16] J. Wang, Y.-c. K. Chen-Wiegart and J. Wang, "In operando tracking phase transformation evolution of lithium iron phosphate with hard X-ray microscopy," Nature Communications, vol. 55, no. 70, pp. 1-10, 2014.

[17] D. Andre, M. Meiler, K. Steiner and C. Wimmer, "Characterization of high-power lithium-ion batteries by electrochemical impedance spectroscopy. I. Experimental Investigation," Journal of Power Sources, vol. 196, no. 12, pp. 5334-5341, 15 June 2011.

[18] S. Buller and M. Thele, "Impedance-based simulation models of supercapacitors and Li-ion batteries for power electronic applications," IEEE Transactions on Industry Applications, vol. 41, no. 3, pp. 742-747, June 2005.

[19] P. Agarwal, M. Orazem and L. Garcia-Rubio, "Measurement Models for Electrochemical Impedance Spectroscopy I. Demonstration of Applicability," Journal of the Electrochemical Society, vol. 139, no. 7, pp. 1917-1927, 1992.

[20] C.-Y. Wang, X. Tang, Y. Zhang, "Cycling Degradation of an automotive LiFePO4 lithium-ion battery," Journal of Power Sources, vol. 196, no. 1, pp. 1513-1520, 2010.

[21] M. Balasubrmanian, H. Lee and A. Moodenbaugh, "Formation of SEl on cycled Lithium-lon Battery Cathodes: Soft X-Ray Absorption Study," Journal of the Electrochemical Society, vol. 5, no. 1, pp. A22-A26, 2001.

[22] J. Vetter, P. Novak and M. Wagner, "Aging mechanisms in lithium-ion batteries," Journal of Power Sources, vol. 147, no. 1-2, pp. 269-281, 2005.

[23] C. Wang, J. Appleby and F. Little, "Electrochemical impedance study of initial lithium ion intercalation into graphite powders," Electrochimica Acta, vol. 46, no. 1, pp. 1793-1813, 2001.

[24] L. Grunes, "Study of the K edges of $3 d$ transition metals in pure and oxide form by x-rayabsorption spectroscopy," Physical Review B, vol. 27, no. 4, pp. 2111-2130, 1983. 
[25] Y-S. Hong, K.S. Ryu, Y.J. Park, M.G. Kim, J.M. Lee, S.H. Chang, "Amorphous $\mathrm{FePO}_{4}$ as $3 \mathrm{~V}$ cathode material for lithium secondary batteries," Journal of Materials Chemistry, vol. 12, no. 1, pp. 18701874, 2002.

[26] Y. Li, F. Gabaly and T. Ferguson, "Current-induced transition from particle-by-particle to concurrent intercalation in phase separating battery electrodes," Nature Materials, vol. 13, no. 1, pp. 1149-1156, 2014.

[27] J. Wang, K. Chen-Wiegart and J. Wang, "In operando tracking phase transformation evolution of lithium iron phosphate with hard X-ray microscopy," Nature Communications, vol. 5, no. 1, pp. 110, 2014.

[28] D. Aurbach, B. Markovsky, A. Shecter and Y. Ein-Eli, "A comparitive study of synthetic graphite and Li electrodes in electrolyte solutions based on ethylene cabonate-dimethyl carbonate mixtures," Journal of the Electrchemical Society, vol. 143, no. 12, pp. 3809-3821, 1996.

[29] S. S. Zhang, "A review on electrolye additives for lithium-ion batteries," Journal of Power Sources, vol. 162, pp. 1379-1394, 2006.

[30] D. N. Wong, D. A. Wetz, J. M. Heinzel and A. N. Mansour, "Degradation characteristics of LiFePO4 cells under ultra-high rate, pulse discharge cycling," Journal of Power Sources, 2015.

[31] J.-S. Shin, C.-H. Han and U.-H. Jung, "Effect of Li2CO3 additive on gas generation in lithium ion batteries," Journal of Power Sources, vol. 109, pp. 47-52, 2002. 